T. Sunada

Nagoya Math. J.

Vol. 57 (1974), 175-200

\title{
NON-LINEAR ELLIPTIC OPERATORS ON A COMPACT MANIFOLD AND AN IMPLICIT FUNCTION THEOREM
}

\author{
TOSHIKAZU SUNADA
}

\section{Introduction}

Many problems in differential and analytic geometry seem to have something to do with the study of non-linear partial differential equations of elliptic type. For instance, the classical Weyl and Minkowski problems for a convex surface have been studied by $\mathrm{H}$. Weyl, H. Lewy, and L. Nirenberg using the iteration method for the construction of solutions of certain non-linear equations of elliptic type (see [6]). Also, M. Kuranishi [3] constructed the effective complete family of deformations of complex analytic structures on a given compact complex manifold as the solution space of another non-linear equation of elliptic type; whereby the basic idea in his work is to apply an implicit function theorem to the non-linear operator of a Banach space, and to construct the bifurcation of solutions explicitly.

In this paper, we will make researches with some geometric structures of the solution spaces of non-linear elliptic systems of partial differential equations defined on a compact manifold, by using the generalized bifurcation method in (infinite dimensional) Fréchet manifolds. The central theme of the present paper is to investigate an Implicit Function Theorem (I.F.T.) for non-linear elliptic operators in the category of the Fréchet topology. In his monograph [9], R. S. Palais has developed a very general culculus for non-linear differential operators on a $C^{\infty}$-manifold from a global point of view. Following the formulation of Palais, we shall deal with operators acting on the space of global cross sections of a $C^{\infty}$-fiber bundle. In the case of opertors acting on smooth functions, namely in the case of those on a trivial line bundle, we have announced our results in [10].

We will now describe the results of the present paper. Let $L$ be a

Received June 24, 1974. 
(non-linear) differential operator from $\Gamma(E)$ to $\Gamma(F)$, where $E$ and $F$ are $C^{\infty}$-fiber bundles on a compact manifold $M$, and $\Gamma(E)$ (resp. $\Gamma(F)$ ) denotes the space of $C^{\infty}$-global cross sections of $E$ (resp. $F$ ). We suppose that $L$ is elliptic at $s \in \Gamma(E)$, namely, its linearization $d_{s} L$ of $L$ at $s \in \Gamma(E)$ by the Gâteaux derivation (it always exists) is a linear elliptic operator from $\Gamma\left(T_{s} E\right)$ to $\Gamma\left(T_{L(s)} F\right)$, where $T_{s}(F)$ (resp. $T_{L(s)}(F)$ ) denotes the vector bundle $s^{-1} T F(E)$ (resp. $L(s)^{-1} T F(F)$ ) induced by $s$ (resp. $L(s)$ ) from $T F(E)$ (resp. $T F(F)$ ), that is known as the vector bundle along the fiber of $E$ (resp. $F$ ). We state in Theorem $1, \S 4$, that the solution space $\mathbb{S}_{s}=$ $\{t \in \Gamma(E) ; L(t)=L(s)\}$ is locally a finite dimensional subset in $\Gamma(E)$ around $s$. To state more precisely, there is an open neighborhood $\mathfrak{U}$ of $s$ in $\Gamma(E)$ with respect to the $C^{\infty}$-topology such that $\mathfrak{S}_{s} \cap \mathfrak{U}$ is diffeomorphic to a locally closed set of the finite dimensional vector space $T_{s}(\widetilde{S})=$ $\left\{v \in \Gamma\left(T_{s} E\right) ; d_{s} L(v)=0\right\}$. This theorem is in part a generalization of a result of J. Moser [4], who proved that, without the assumption of ellipticity, the solution space is of zero-dimension when $d_{s} L$ is injective. Later, M. Ise proposed the conjecture that the local dimension of $\mathfrak{S}_{s}$ at $s$ is not greater than the dimension of $T_{s}(\mathbb{S})$ in the case where $E$ and $F$ are trivial line bundles. Our result is in a sense a verification of this conjecture.

In order to prove Theorem 1 , we have to construct the bifurccation operator $\Phi$ related to the operator $L$, based on the Hodge-Kodaira decomposition, and show that $\Phi$ endows a local diffeomorphism. Although it is easy to show that the linearization of $\Phi$ is an isomorphism of certain Fréchet spaces, we can not immediately apply the usual I.F.T. to the operator $\Phi$, because it does not hold in general for Fréchet space. For this reason, by utilizing the so-called I.L.H.-method introduced in H. Ômori [7] and the regularity of solutions of a non-linear elliptir equation (see A. Douglis-L. Nirenberg [1]), we prove a modified Implicit Function Theorem in certain Fréchet spaces with the $C^{\infty}$-topology, which is a unified method of non-linear global analysis and infinite dimensional geometry (see [2], [5]).

The contents of this paper are as follows: To begin with, in $\S 1$, we review of the manifold structure of the space of global sections of $C^{\infty}$-fiber bundles and the notion of non-linear elliptic operators, following Palais [9]. In $\S 2$, we will explain two simple examples of non-linear elliptic (overdetermined elliptic) operators. Some general results about 
linear elliptic operators with $C^{\infty}$-coefficients, for instance, the classical theory of Hodge-Kodaira's decomposition, which we shall utilize in the later sections, are summarized in $\S 3$. In $\S 4$, we state the main theorem of this paper, and apply the theorem to the examples given in $\S 2$. We also apply in $\S 5$ a modified implicit function theorem to the study of the solution space of non-linear equations. Using the results in $\S 3$, we prove an I.F.T. for elliptic operators in $\S 6$. The proof is based mainly upon the regularity of solutions. Finally, in $\S 7$, we give a more precise result for the solution space under an additional assumption. In the course of our arguments there, we shall need the notion of the minimal elements.

Interesting examples and applications to differential and analytic geometry will be discussed in a forthcoming paper.

The author is grateful to Professor Mikio Ise for suggesting the present problem and for his advices in the course of preparation of this paper.

\section{§ 1. Review of the manifold structure of the space of global sections}

We now fix our notations and recall some results which will be used later. Specifically, we define the (non-linear) differential operators acting on the space of cross sections of a $C^{\infty}$-fiber bundle, using the notion of the jet bundles and the jet extension mapping. As for the definitions and the results summerized here, we refer to Palais [8] as a standard reference.

Let $M$ be a compact $n$-dimensional $C^{\infty}$-manifold without boundary, and let $\pi: E \rightarrow M$ be a $C^{\infty}$-fiber bundle over $M$. For an integer $k \geqq n / 2+1$, we get a $C^{\infty}$-Hilbert manifold $W^{k}(E)$, called the Sobolev manifold of degree $k$, defined as the set of all global cross sections of $E$ whose (distributional) derivatives of order $\leqq k$ with respect to any local coordinates are square integrable.

Let $\Gamma^{h}(E)$ denote the space of sections of $E$ of class $C^{h}$. This is a Banach manifold under so-called $C^{k}$ topology. The Sobolev embedding theorem states that if $h \geqq 0$ and $k>(n / 2)+h$, then $W^{k}(E) \subset \Gamma^{h}(E)$ and the inclusion mapping is continuous.

We shall define a differentiable structure for $\Gamma(E)=\Gamma^{\infty}(E)$, the space of global $C^{\infty}$-cross sections of $E$, by expressing the $C^{\infty}$-topology as the inverse limit of the topologies of $W^{k}(E)$ : 


$$
\Gamma(E)=\lim _{\longleftarrow} W^{k}(E)=\bigcap_{k>n / 2} W^{k}(E) .
$$

Then the Fréchet manifold $\Gamma(E)$ is called an I.L.H.-manifold (the inverse limit of Hilbert manifolds; see [7]).

From the local triviality of fiber bundle $\pi: E \rightarrow X$, it follows that the projection $\pi$ is a submersion (namely, its differential $d \pi ; T E \rightarrow T X$ is surjective), whence that $\operatorname{Ker}(d \pi)$ is a vector subbundle of the tangent bundle $T E$ of $E$. This vector bundle over $E$ will be denoted by $T F(E)$, and is usually called the tangent bundle along the fiber of $E$. If $s \in \Gamma(E)$, then the induced bundle $s^{-1} T F(E)$ by $s$ is a vector bundle over $M$ which we denote simply by $T_{s}(E)$. Then, the space of cross section, $\Gamma\left(T_{s} E\right)$, plays a role of the tangent space at $s$ of the Fréchet manifold $\Gamma(E)$.

For any continuous section $s \in \Gamma^{\circ}(E)$, we define a vector bundle neighborhood of $s$ in $E$ by a vector bundle $\xi$ over $M$, such that $\xi$ is an open subbundle of $E$ and $s \in \Gamma^{0}(\xi)$. Then, for any fiber bundle $E, s \in \Gamma^{0}(E)$, and any neighborhood $U$ of $s(M)$ in $E$, there exists a vector bundle neighborhood $\xi$ of $s$ in $E$ with $\xi \subseteq U$. Moreover, if $s \in \Gamma(E)$ we can choose $\xi$ so that $s$ is the zero section of $\xi$; in this case $\xi$ is clearly isomorphic to $T_{s}(E)$.

Using the vector bundle neighborhoods, we have a covering

$$
W^{k}(E)=\bigcup_{\xi} W^{k}(\xi),
$$

where in the union $\xi$ runs over all open vector bundle neighborhoods of $E$ and $W^{k}(\xi)$ is an open submanifold of $W^{k}(E)$.

Next we shall define (non-linear) differential operators acting on the space of cross sections of a fiber bundle. For this purpose, we construct first the $m$-jet bundle $J^{m} E$ of $E$, as follows: For a given $e \in E$ with $\pi(e)=x$ and local cross sections $s_{1}, s_{2}$ of $E$ defined around $x$ with $s_{1}(x)=s_{2}(x)=e$, we choose a chart around $x$ in $M$, a local trivialization of $E$ around $x$, and a chart around $e$ in the fiber $E_{x}=\pi^{-1}(x)$ respectively; under these conditions, if the $m$-th order Taylor expansions of $s_{1}$ and $s_{2}$ coincide each other, we say that $s_{1}$ and $s_{2}$ have the same $m$-jet at $x$. This defines an equivalence rclation on the set of local cross sections $s$ of $E$ around $x$ with $s(x)=e$. The set of equivalence classes is denoted by $J^{m} E_{e}$ and the equivalence class containing $s$ is denoted by $j_{m}(s)_{x}$. Put $J_{0}^{m}(E)=\bigcup_{e \in E} J^{m} E_{e}$ and $\pi_{0}^{(m)}: J_{0}^{m}(E) \rightarrow E$ denotes the projection given by 
$\pi_{0}^{(m)}\left(J_{0}^{m} E_{e}\right)=e$. We define a $C^{\infty}$-fiber bundle over $M$

$$
\pi^{(m)}: J^{m} E \rightarrow M,
$$

whose total space is $J_{0}^{m} E$ and whose projection is the composition $\pi^{(m)}$ $=\pi \circ \pi_{0}^{(m)}$. Then bundle $J^{m} E$ is called the $m$-jet bundle of $E$, and the mapping $j_{m}: \Gamma(E) \rightarrow \Gamma\left(J^{m} E\right)$ defined by $j_{m}(s)(x)=j_{m}(s)_{x}$ is called the $m$ jet extension mapping.

Now let $F$ be another $C^{\infty}$-fiber bundle over $M$. Then a mapping $L: \Gamma(E) \rightarrow \Gamma(F)$ will be called a (non-linear) differential operator of order $m$ from $E$ to $F$ if it can be factored as

$$
\Gamma(E) \stackrel{j_{m}}{\longrightarrow} \Gamma\left(J^{m} E\right) \stackrel{T_{*}}{\longrightarrow} \Gamma(F),
$$

where $T: J^{m} E \rightarrow F$ is a $C^{\infty}$-fiber bundle mapping over $M$.

In the case of the operator $L$ acting on smooth functions, a local expression of $L$ with respect to local coordinates is represented as follows: Choose the local coordinates $x_{1}, \cdots, x_{n}$ in a coordinates neighborhood $U$ in $M$. In this case, the jet extension mapping is given by the derivation of order $\leqq m: C^{\infty}(U) \rightarrow C^{\infty}(U)^{N}$, that is furnished by $u \mapsto\left\{D^{\alpha} u\right\}_{|\alpha| \leqq m}$, where $N$ denotes the number of multi-indices $\alpha=\left(\alpha_{1}, \cdots, \alpha_{n}\right)$ with $|\alpha|=\sum \alpha_{i} \leqq m$, and $D^{\alpha}$ denotes the partial derivation $\partial^{\alpha_{1}} / \partial x_{1}^{\alpha_{1}} \cdots \partial^{\alpha_{n}} / \partial x_{n}^{\alpha_{n}}$ as usual. Further, the fiber bundle mapping: $U \times \boldsymbol{R}^{N} \rightarrow U \times \boldsymbol{R}$ is given by an element of $C^{\infty}\left(U \times R^{N}\right)$, say $F\left(x, y^{(\alpha)}\right)$. Then the operator: $C^{\infty}(U) \rightarrow C^{\infty}(U)$ induced by $L$ is of the form:

$$
L(u)=F\left(x, D^{\alpha} u\right) .
$$

Every (non-linear) differential operator $L$ of order $m$ can be extended to a $C^{\infty}$-mapping of the Hilbert manifolds:

$$
L^{(k)}: W^{k}(E) \rightarrow W^{k-m}(F)
$$

for $k>(n / 2)+m$. Then the Fréchet derivative $d_{s} L^{(k)}$ of $L^{(k)}$ at $s \in \Gamma(E)$ is a linear mapping of $T_{s}\left(W^{k} E\right)$ into $T_{L(s)}\left(W^{k-m} F\right)$, where $T_{s}\left(W^{k} E\right)$ (resp. $T_{L(s)}\left(W^{k-m} F\right)$ ) denotes the tangent space of Hilbert manifold $W^{k}(E)$ at $s$ (resp. $W^{k-m}(F)$ at $L(s)$ ). Now we have canonical identification:

$$
\begin{aligned}
& T_{s}\left(W^{k} E\right)=W^{k}\left(T_{s} E\right), \\
& T_{L(s)}\left(W^{k-m} F^{\prime}\right)=W^{k-m}\left(T_{L_{(s)}} F\right),
\end{aligned}
$$

and have a linear differential operator of order $m$ : 


$$
d_{s} L: \Gamma\left(T_{s} E\right) \rightarrow \Gamma\left(T_{L(s)} F\right),
$$

which is called the linearization of $L$ at $s$, with the property $\left(d_{s} L\right)^{(k)}=$ $d_{s} L^{(k)}$; namely, the extension of linearization of $L$ coincides with the Fréchet derivative of the extension. We can also get $d_{s} L$ by Gâteaux derivation; more precisely, let $\xi$ be a vector bundle neighborhood of $s$ in $E$ and $\zeta$ that of $L(s)$ in $F$. Then for $\Gamma(\xi)$

$$
\frac{1}{t}(L(s+t u)-L(s))
$$

converges in the $C^{\infty}$-topology to $d_{s} L(u)$ as $t \rightarrow 0$. Therefore, if $L$ has a local expression as above, its linearization can be expressed by

$$
d_{s} L(u)=\left.\sum_{|\alpha| \leqq m} \frac{\partial F\left(x, y^{(\alpha)}\right)}{\partial y^{(\alpha)}}\right|_{y(\alpha)=D^{\alpha} s} D^{\alpha} u
$$

Since the operator $d_{s} L$ is a linear differential operator of order $m$, we can consider the leading symbol $\sigma_{m}\left(d_{s} L\right)$ in a usual manner. Namely this is a bundle homomorphism

$$
\sigma_{m}\left(d_{s} L\right): p^{-1} T_{s} E \rightarrow p^{-1} T_{L(s)} F
$$

over the cotangent space $T^{*} M$ of $M$ ( $p$ denotes the projection $\left.T^{*} M \rightarrow M\right)$. Here, we define the ellipticity of non-linear differential operators, namely $L$ will be called elliptic (resp. overdetermined elliptic) at $s$ if $\sigma_{m}\left(d_{s} L\right)$ is an isomorphism (resp. monomorphism) outside the zero section of $T^{*} M$, that is to say, if $d_{s} L$ is a linear elliptic (resp. overdetermined elliptic) operator of $m$-th order in the usual sense. We notice that if $L$ is elliptic at $s$, then there exists a neighborhood $\mathfrak{U}$ of $s$ such that for any $u \in \mathfrak{H}, d_{u} L$ is elliptic. This is a simple consequence of the definition of ellipticity.

\section{§ 2. Examples}

Firstly, we give a very simple example of non-linear elliptic operator acting on complex-valued $C^{\infty}$-functions. We let $\boldsymbol{R}$ denote the real numbers, $Z$ the integers, and $T^{n}=R^{n} /(2 \pi Z)^{n}$ the flat torus with the natural flat metric, where $(2 \pi Z)^{n}=2 \pi Z \times \cdots \times 2 \pi Z$ denotes the lattice with the side of length $2 \pi$. The point of $T^{n}$ is usually represented by the angular parameters $x_{1}, \cdots, x_{n}\left(0 \leqq x_{i}<2 \pi\right)$. We consider a non-linear elliptic operator $L$ defined as 


$$
L u=\Delta u+F(u)
$$

where $\Delta$ stands for the usual Laplacian: $\left(\partial^{2} / \partial x_{1}^{2}\right)+\cdots+\left(\partial^{2} / \partial x_{n}^{2}\right)$, and $F: C \rightarrow C$ an analytic function. Then, the linearization of $L$ at $f \in C^{\infty}(M)$ is given by

$$
d_{f} L(u)=\Delta u+F^{\prime}(f) u .
$$

Here, $F^{\prime}$ is the usual derivative of $F$. Hence, for all $f \in C^{\infty}(M)$, the operator $L$ is elliptic at $f$. In particular, in a special case where $F(u)$ $=u+u^{2}$, and $f \equiv 0$, we have

$$
d_{0} L(u)=\Delta u+u^{(*)}
$$

We notice that the dimension of $\operatorname{Ker} d_{0} L$ over $C$ is $2 n .^{(* *)}$

Secondarily, we consider a more geometric example. Let $(M, g)$ be a compact Riemannian manifold with the metric $g$, and let $\pi: M \times M \rightarrow M$ be the proposition on its first factor: $\pi(x, y)=x$. Then, we may consider $M \times M$ as a trivial fiber bundle over $M$ with projection $\pi$, and the space of cross sections, $\Gamma(M \times M)$, can be identified with $C^{\infty}(M, M)$, which is the space of $C^{\infty}$-mappings of $M$ to $M$, by putting $s(x)=(x, \varphi(x))$ $\left(\varphi \in C^{\infty}(M \times M)\right)$. We here denote by $S^{2} M=S^{2}\left(T^{*} M\right)$ the symmetric covariant tensor bundle of degree 2 . We can then define the operator

$$
L: \Gamma(M \times M) \rightarrow \Gamma\left(S^{2} M\right)
$$

by putting $L(\varphi)=\varphi^{*} g$ for $\varphi \in \Gamma(M \times M)=C^{\infty}(M, M)$, where $\varphi^{*} g$ is the tensor field induced from $g$ by $\varphi$ (notice that $g \in \Gamma\left(S^{2} M\right)$ ).

Now, to see that $L$ is a differential operator of first order, we shall write down a local expression of $L$; let $g_{i j}$ denote the components of $g$ with respect to a local coordinate system $\left(x_{1}, \cdots, x_{n}\right)$ around $x$; namely, $g_{i j}=g\left(\partial / \partial x_{i}, \partial / \partial x_{j}\right)$, and also let $\left(y_{1}, \cdots, y_{n}\right)$ be the one around $\varphi(x)$. Then, we have

$$
\left(\varphi^{*} g\right)_{k \ell}=\sum_{i, j} g_{i j}(\varphi(x)) \frac{\partial \varphi_{i}}{\partial x_{k}} \frac{\partial \varphi_{j}}{\partial x_{\ell}},
$$

where $\varphi_{i}=y_{i} \circ \varphi$. Thus, we see that $L$ is in fact a differential operator of first order.

Next, we determine the linearization of $L$, at the identity mapping Id $\in C^{\infty}(M, M)$. For this sake, we identify $T_{\mathrm{Id}}(M \times M)$ with $T M$ as fol-

(*) This case was suggested by K. Masuda (see also §4).

(**) In the real case, the solution $u \equiv 0$ is isolated in the solution space of $L u=0$. 
lows: $\quad T_{\mathrm{Id}}(M \times M)=(\mathrm{Id})^{-1} T F(M \times M)=(\mathrm{Id})^{-1} \pi^{-1} T M=T M$. We can then prove:

LEMMA 1. Under the identification of $T_{\mathrm{Id}}(M \times M)$ with $T M$ mensioned above, we have

$$
d_{\mathrm{Id}} L(X)=\mathscr{L}_{x} g,
$$

where $X$ denotes a $C^{\infty}$-vector field, and $\mathscr{L}_{x}$ denotes the Lie derivative with respect to $X$.

Proof. Define now the mapping $\varphi: T M \rightarrow M \times M$ by

$$
\varphi(v)=(p(v), \exp v),
$$

where $p: T M \rightarrow M$ denotes the bundle projection and exp:TM $\rightarrow M$ denotes the exponential mapping with respect to the Riemannian metric $g$. As is well-known, for a small positive number $\varepsilon, \varphi$ defines the bundle isomorphism of $T_{\varepsilon}(M)=\{v \in T M ;\|v\|<\varepsilon\}$ onto an open subbundle of $M \times M$ containing the diagonal $\Delta(M) \subset M \times M$ such that $\varphi\left(0_{x}\right)=(x, x)$ and the linearization $d_{0} \varphi: \Gamma(T M) \rightarrow \Gamma(T M)$ at the zero-section of $T M$ is the identity mapping of $\Gamma(T M)$. Hence, it is enough to show that

$$
d_{0}(L \circ \varphi)(X)=\mathscr{L}_{x} g .
$$

We take a local chart $\left(U ; x_{1}, \cdots, x_{n}\right)$ of $M$, and let $X=\sum_{i} \xi^{i}\left(\partial / \partial x_{i}\right)$ be a $C^{\infty}$-vector field. For a small $t$, we put

$$
x_{i}(\exp t X)=\varphi^{i}\left(x, t \xi^{1}(x), \cdots, t \xi^{n}(x)\right) .
$$

Then, we have

$$
\begin{aligned}
d_{0}(L \circ \varphi)(X)= & \lim _{t \rightarrow 0} \frac{1}{t}\left(\varphi(t X)^{*} g-g\right)=\left\{\left.\frac{d}{d t}\left(\sum_{i, j} g_{i j}(\varphi(t X)) \frac{\partial \varphi^{i}}{\partial x_{\ell}} \frac{\partial \varphi^{j}}{\partial x_{k}}\right)\right|_{t=0}\right\}_{k \ell} \\
= & \left\{\left.\frac{d}{d t}(\varphi(x, t \xi))\right|_{t=0}+\left.\sum_{i} g_{i k}(x) \frac{d}{d t}\left(\frac{\partial \varphi^{i}}{\partial x_{\ell}}\right)\right|_{t=0}\right. \\
& \left.+\left.\sum_{j} g_{\ell j}(x) \frac{d}{d t}\left(\frac{\partial \varphi^{j}}{\partial x_{k}}\right)\right|_{t=0}\right\}_{k \ell}
\end{aligned}
$$

By the way, it is obvious that $\left.(d / d t) \varphi^{i}(x, t \xi)\right|_{t=0}=\xi^{i}$; whence we obtain

$$
d_{\mathrm{Id}} L(X)=d_{0}(L \circ \varphi)(X)=\left\{\sum_{h} \frac{\partial g_{k \ell}}{\partial x_{h}} \xi^{h}+\sum_{i} g_{i k} \frac{\partial \xi^{i}}{\partial x_{\ell}}+\sum_{j} g_{\ell j} \frac{\partial \xi^{j}}{\partial x_{k}}\right\}_{k \ell}=\mathscr{L}_{x} g
$$

This completes the proof. 
REMARK. A vector field $X$ satisfying that $\mathscr{L}_{x} g=0$ is called a Killing vector field.

The operator $L$ defined above is not elliptic at $\operatorname{Id} \in C^{\infty}(M, M)$. However, we have from [8]

LEMMA 2. The leading symbol of $d_{\mathrm{Id}} L$ is injective (namely, $d_{\mathrm{Id}} L$ is overdetermined elliptic).

\section{§ 3. Summary of the Hodge-Kodaira theory for linear elliptic operators}

We will now summarize the Hodge-Kodaira theory for linear elliptic operators on a compact manifold which we shall need in later sections. The proofs will be found in Palais [8].

In what follows, $E$ and $F$ will denote $C^{\infty}$ vector bundles over $M$, and we consider a linear elliptic operator of $m$-th order:

$$
D: \Gamma(E) \rightarrow \Gamma(F)
$$

Furthermore, we assume once and for all that there is given on $M$ a Riemannian metric $g=\left(g_{i j}\right)$ and a hermitian metric $\langle,\rangle_{E}$ in $F$ (resp. $\langle,\rangle_{F}$ in $F^{\prime}$ ). We denote by $d M(g)=\sqrt{\operatorname{det}\left(g_{i j}\right)} d x_{1} \wedge \cdots \wedge d x_{n}$ the volume element of $M$ with respect to $g$, and by $(s, r)_{E}$ the $L^{2}$-inner product on $\Gamma(E)$ (resp. $(t, u)_{F}$ on $\Gamma(F)$ ), which is defined by

$$
(s, r)_{E}=\int_{M}\langle s, r\rangle_{E} d M(g), \quad(t, u)_{F}=\int_{M}\langle t, u\rangle_{F} d M(g)
$$

Let $D^{*}$ now denote the formal adjoint operator of $D$ with respect to these inner products, that is, $D^{*}$ is given by

$$
(D s, t)_{F}=\left(s, D^{*} t\right)_{E}
$$

for every $s \in \Gamma(E), t \in \Gamma(F)$. In fact, $D^{*}$ becomes also an elliptic operator of $m$-th order.

As before, we denote by $J^{k} E$ (resp. $J^{k} F$ ) the $k$-jet bundle of $E$ (resp. $F)$ and by $j_{k}: \Gamma(E) \rightarrow \Gamma\left(J^{k} E\right)$ the $k$-jet extension mapping. In this case, $J^{k}(E)$ is a vector bundle and $j_{k}$ is a linear mapping. Introducing a metric in $J^{k} E$ (resp. in $J^{k} F$ ), we can define the Sobolev inner product of degree $k$ as follows: 


$$
\begin{aligned}
& (s, r)_{E, k}=\int_{M}\left\langle j_{k} s, j_{k} r\right\rangle_{J^{k} E} d M(g), \\
& (t, u)_{F, k}=\int_{M}\left\langle j_{k} t, j_{k} u\right\rangle_{J^{k} F} d M(g) .
\end{aligned}
$$

We put further

$$
\|s\|_{*, k}^{2}=(s, s)_{*, k} .
$$

Then, by the Sobolev space $W^{k}(*)$ of degree $k$, we mean the completion of $\Gamma(*)$ with respect to the Sobolev norm $\|\cdot\|_{*, k}(*$ denotes $E$ or $F)$. We would like to point out here that the Sobolev space $W^{k}(E)$ coincides with the Sobolev manifold introduced in $\S 1$ in the case where $\pi: E \rightarrow M$ is a vector bundle.

From the definition of the Sobolev space, we have a natural inclusion: $W^{k+1} \subset W^{k}$. Rellich's lemma asserts that the inclusion: $W^{k+1} \subset W^{k}$ is a compact operator of Hilbert spaces. Thus, we get a discrete chain of Hilbert spaces:

$$
W^{0} \supset W^{1} \supset \cdots \supset W^{k} \supset \cdots
$$

such that $\lim W^{k}=\cap W^{k}=\Gamma$.

The Sobolev inner product defined as above, however, seems to be inconvenient to investigate the properties of elliptic operators. Therefore, we will introduce another inner product of $\Gamma(*)$ which is equivalent to the Sobolev norm: We put first

$$
\square_{E}=D^{*} D, \quad \square_{F}=D D^{*} ;
$$

$\square_{E}$ (resp. $\square_{F}$ ) is a formally self-adjoint strongly elliptic operator of order $2 m$ (called the Laplacian associated with $D$ ). We define then the inner products in $\Gamma(E)$ and $\Gamma(F)$ related to the Laplacian $\square *$ by the following :

$$
\begin{aligned}
& {[s, r]_{E, k}=\left(\left(\square \square_{E}+1\right)^{k} s, r\right)_{E},} \\
& {[t, u]_{F, k}=\left(\left(\square_{F}+1\right)^{k} t, u\right)_{F},}
\end{aligned}
$$

where $k$ is a non-negative integer and $s, r \in \Gamma(E), t, u \in \Gamma(F)$. Denote further by $H^{k}(E)$ (resp. by $H^{k}\left(F^{\prime}\right)$ ) the completions of $\Gamma(E)$ (resp. of $\dot{\Gamma}\left(F^{\prime}\right)$ ) with respect to the inner product $[,]_{E, k}\left(\operatorname{resp} .[,]_{F, k}\right)$. It is clear that $[,]_{*, 0}=(,)_{*}$. 
Hereafter, $\square$ (resp. $H^{k}$ and $\Gamma$ represents either $\square_{E}$ or $\square_{F}$ (resp. $H^{k}(E)$ or $H^{k}\left(F^{\prime}\right)$, and $\Gamma(E)$ or $\Gamma(F)$ ), for the brevity. Some well known properties of the Laplacian $\square$ which we will need are as follows:

(1) (Gårding inequality) For any $s \in \Gamma$, it holds: $(\square s, s) \geqq C_{0}\|s\|_{m}^{2}$ - $\|s\|_{0}^{2}$, where $\|s\|_{m}$ denotes the Sobolev norm of $s \in \Gamma$ of degree $m$.

(2) (A-priori estimate), $\|s\|_{2 m+k} \leqq C_{1}\left(\|(\square+1) s\|_{k}+\|s\|_{0}\right) . \quad\left(C_{0}\right.$ and $C_{1}$ are constants not depending on $s \in \Gamma$ ).

Lemma 3. $H^{k}$ is isomorphic to the Sobolev space $W^{k m}$ as Banach spaces.

Proof. By virtue of the Gårding inequality for the Laplacian $\square$, we have

$$
((\square+1) s, s) \geqq C_{0}\|s\|_{m}^{2} .
$$

On the other hand, if we make use of (1) and (2), one has a inequality

$$
\|(\square+1) s\|_{k} \geqq \text { const. }\|s\|_{2 m+k}
$$

for all $k \geqq 0$. For, from the Gårding inequality, it follows that

$$
\|(\square+1) s\|_{0}\|s\|_{0} \geqq((\square+1) s, s)_{0} \geqq C_{0}\|s\|_{m}^{2} \geqq \text { const. }\|s\|_{m}\|s\|_{0},
$$

which proves that $\|(\square+1) s\|_{0} \geqq$ const. $\|s\|_{m}$. Combining this inequality and (2), we have

$$
\begin{aligned}
\|s\|_{2 m+k} & \leqq \text { const. }\left(\|(\square+1) s\|_{k}+\|s\|_{0}\right) \leqq \text { const. }\left(\|(\square+1) s\|_{k}+\|s\|_{m}\right) \\
& \leqq \text { const. }\left(\|(\square+1) s\|_{k}+\text { const. }\|(\square+1) s\|_{0}\right) \leqq \text { const. }\|(\square+1) s\|_{k} .
\end{aligned}
$$

This proves (3).

We now claim that the norm $[s]_{k}=[s, s]_{k}^{1 / 2}$ is equivalent to the Sobolev norm $\|s\|_{m k}$. First we consider the case when $k$ is even $(=2 \ell)$. The above inequality (3) yeilds

$$
\begin{aligned}
{[s, s]_{k} } & =\left((\square+1)^{k} s, s\right)=\left((\square+1)^{\ell} s,(\square+1)^{\ell} s\right) \\
& \geqq \text { const. }\|s\|_{2 e m}^{2}=\text { const. }\|s\|_{k m}^{2} .
\end{aligned}
$$

While it is clear that

$$
[s, s]_{k} \leqq \text { const. }\|s\|_{k m}^{2},
$$

since $(\square+1)^{\ell}$ is a differential operator of order $2 \ell m$. Hence, we can conclude that $[s, s]_{k}$ is equivalent to $\|s\|_{k m}^{2}$ when $k$ is even. Next, when $k$ is odd $(=2 \ell+1)$, we have similarly 


$$
\begin{aligned}
{[s, s]_{k} } & =\left((\square+1)(\square+1)^{\ell} s,(\square+1)^{\ell} s\right) \geqq \text { const. }\left\|(\square+1)^{\ell} s\right\|_{m}^{2} \\
& \geqq \text { const. }\|s\|_{m+2 \ell m}^{2}=\text { const. }\|s\|_{k m}^{2} .
\end{aligned}
$$

Furthermore, we have

$$
[s, s]_{k} \leqq \text { const. }\left\|(\square+1)^{\ell} s\right\|_{m}^{2} \leqq \text { const. }\|s\|_{k m}^{2},
$$

where we use the inequality: $((\square+1) s, s) \leqq\|s\|_{m}^{2}$. Thus we get the same conclusion in this case, too. This completes the proof of the lemma.

REMARK. According to the above lemma, we know that $D$ can be extended to $D^{(k)}: H^{k}(E) \rightarrow H^{k-1}(F)$.

Now, we introduce for every $\lambda \in C$ the space

$$
\Gamma_{\lambda}=\{s \in \Gamma ; \square s=\lambda s] .
$$

If $\Gamma_{\lambda} \neq(0)$, we call $\lambda$ an eigen-value of $\square$. Since $(\square s, s) \geqq 0$, all eigenvalue are non-negative. We can now establish the following basic properties of eigen-value of Laplacian:

Proposition. (i) The set of eigen-value of $\square$ is discrete:

$$
0 \leqq \lambda_{0}<\lambda_{1}<\lambda_{2}<\cdots \rightarrow \infty \text {. }
$$

(ii) The eigen-space $\Gamma_{\lambda}$ is a finite dimensional vector space for any eigen-value $\lambda$.

(iii) We have a $H^{k}$-orthogonal decomposition

$$
H^{k}=\sum_{\lambda} \oplus \Gamma_{\lambda}
$$

where the summation runs over all eigen-value of $\square$.

(iv) The decomposition in (iii) is orthogonal with respect to the $L^{2}$ inner product.

Proof. It is first to be noticed that the operator $\square+1$ has the abstruct inverse operator $A$ (namely, $A(\square+1)=(\square+1) A=\mathrm{Id}$ ). In fact, for $t \in \Gamma$, the operator $s \rightarrow(s, t)\left(s \in H^{\prime}\right)$ is bounded with respect to the $H^{1}$-topology. Hence, by Riesz's representation theorem, there is $u \in H^{1}$ such that $[s, u]_{1}=(s, t)$. In particular, for any $s \in \Gamma$, we have

$$
((\square+1) s, u)=(s, t) \text {. }
$$

This implies that $(\square+1) u=t$. In view of hypoellipticity of elliptic operator $\square+1$ (it is due to the $a$-priori estimate), if follows that $u \in \Gamma$. 
The uniqueness of $u$ with $(\square+1) u=t$ is clear. Therefore, $\square+1$ has the inverse $A$. Moreover, from the definition of inner product $[,]_{k}$, we have

$$
\begin{aligned}
& {[A s, t]_{k}=[s, t]_{k-1}=[s, A t]_{k},} \\
& {[A s]_{k}=[s]_{k-2},}
\end{aligned}
$$

and so $A$ has the extension $A^{(k)}: H^{k} \rightarrow H^{k+2}$. By the way, we know that the inclusion: $H^{k+2} \rightarrow H^{k}$ is a compact operator by Rellich's lemma. These imply that $A\left(=A^{(k)}\right)$ is a self adjoint compact operator of $H^{k}$. Hence, it follows from the Riesz theorem for the self adjoint compact operators of Hilbert spaces that, for any given $\lambda \in R, E_{\lambda}(A)=\left\{s \in H^{k}\right.$; $A s=\lambda s\}$ are mutually orthogonal subspaces of $H^{k}$, and $H^{k}$ is their Hilbert space direct sum. Moreover, the spaces $E_{\lambda}(A)$ are finitely dimensional, and the set $\left\{\lambda \in R ; E_{\lambda}(A) \neq(0)\right\}$ of eigenvalues of $A$ has no limit points possibly except zero. If $s \in E_{\mu}(A)$, then $A s=\mu s$ and this means that $\mu(\square+1) s=s$. From the hypoellipticity of $\square+1$, we have $s \in \Gamma_{(1 / \mu)-1}$. In other words, this implies that

$$
\Gamma_{\lambda}=E_{1 /(\lambda+1)}(A) .
$$

This gives the proof of (i), (ii), and (iii).

Now we prove (iv). It suffices to show that the orthogonal projection $\pi_{\lambda}^{k}$ onto $E_{\lambda}$ in $H^{k}$ coincides with $\pi_{\lambda}^{0}$. For this purpose, we take $s \in E_{\lambda}(A)$ and $t \in H^{k}$. Since $[A s, t]_{k}=[s, t]_{k-1}$, we have $\lambda[s, t]_{k}=[s, t]_{k-1}$. This implies that if $\left(s_{1}, \cdots, s_{r}\right)$ are the orthonormal basis of $E_{\lambda}$ in $H^{k}$, then $\left((1 / \sqrt{\lambda}) s_{1}, \cdots,(1 / \sqrt{\lambda}) s_{r}\right)$ are the orthonormal basis for $E_{\lambda}$ in $H^{k-1}$. Let $\pi_{\lambda}^{k}: H^{k} \rightarrow E_{\lambda}$ be the orthogonal projection in $H^{k}$, defined by

$$
\pi_{\lambda}^{k}(t)=\sum_{i=1}^{r}\left[t, s_{i}\right]_{k} s_{i}
$$

for $t \in H^{k}$. Then, we have the equality:

$$
\begin{aligned}
\pi_{\lambda}^{k-1}(t) & =\sum_{i=1}^{r}\left[t, \frac{1}{\sqrt{\lambda}} s_{i}\right]_{k-1} \frac{1}{\sqrt{\lambda}} s_{i}=\sum_{i=1}^{r} \frac{1}{\lambda}\left[t, s_{i}\right]_{k-1} s_{i} \\
& =\sum_{i=1}^{r}\left[t, s_{i}\right]_{k} s_{i}=\pi_{\lambda}^{k}(t) .
\end{aligned}
$$

This implies, in particular, that $\pi_{\lambda}^{k}=\pi_{\lambda}^{0}$. q.e.d.

We put $P_{\lambda}^{k}=\pi_{1 /(\lambda+1)}^{k}: H^{k} \rightarrow \Gamma_{\lambda}$, and we call in particular $H=P_{0}^{0}$ the harmonic projection associated with the Laplacian 
Corollary 1. For any $s \in \Gamma$, the Fourier expansion

$$
\sum_{\lambda} P_{\lambda}^{0} s=\sum_{\lambda} P_{\lambda}^{k} s
$$

converges in $C^{\infty}$-topology of $\Gamma$ to $s$.

CoRollary 2 (Hodge-Kodaira). There is a operator $G: \Gamma \rightarrow \Gamma$, called the Green operator, such that

$$
s=H s+\square G s=H s+G \square s
$$

for $s \in \Gamma$.

Proof. We put

$$
G=\sum_{\lambda>0} \frac{1}{\lambda} P_{\lambda}^{0}
$$

Since $\square P_{\lambda}^{0}=\lambda P_{\lambda}^{0}$, we have

$$
s=\sum_{\lambda \geq 0} P_{\lambda}^{0} s=P_{0}^{0} s+\sum_{\lambda>0} P_{\lambda}^{0} s=H s+\square \sum_{\lambda>0} \frac{1}{\lambda} P_{\lambda}^{\prime} s=H s+\square G s . \quad \text { q.e.d. }
$$

LEMMA 4. $[G s]_{k+2} \leqq$ const. $[s]_{k}$. Especially, G has a extension $G^{(k)}: H^{k}$ $\rightarrow H^{k+2}$.

Proof. By the definition of $G$, we have

$$
\begin{aligned}
{[G s]_{k+2}^{2} } & =\left[\sum_{\lambda>0} \frac{1}{\lambda} P_{\lambda} s\right]_{k+2}^{2}=\sum_{\lambda>0} \frac{1}{\lambda^{2}}\left[P_{\lambda} s\right]_{k+2}^{2} \leqq \text { const. } \sum_{\lambda>0}\left[P_{\lambda} s\right]_{k}^{2} \\
& =\text { const. }\left([s]_{k}^{2}-[H s]_{k}^{2}\right) \leqq \text { const. }[s]_{k}^{2}
\end{aligned}
$$

where we use the fact $(\lambda+1)^{2}\left[P_{\lambda} s\right]_{k}^{2}=\left[P_{\lambda} s\right]_{k+2}^{2}$.

q.e.d.

Corollary. $\operatorname{Im}\left(D^{*}\right)^{(k+1)} \quad\left(\right.$ resp. $\left.\operatorname{Im} D^{(k+1)}\right)$ is a closed subspace in $H^{k}(E)$ (resp. in $H^{k}(F)$ ).

Proof. Since $H^{k}(E)=\Gamma_{0}(E) \oplus\left(\sum_{\lambda>0} \oplus \Gamma_{\lambda}(E)\right)$, it is enough to show that $\operatorname{Im}\left(D^{*}\right)^{(k+1)}=\sum_{\lambda>0} \oplus \Gamma_{\lambda}(E)$. We take $s=\sum_{\lambda>0} P_{\lambda}^{k} s \in \sum_{\lambda>0} \oplus \Gamma_{\lambda}(E)$. Then, we have

$$
s=\square^{(k+2)} G^{(k)} s=\left(D^{*}\right)^{(k+1)} D^{(k+2)} G^{(k)} s \in \operatorname{Im}\left(D^{*}\right)^{(k+1)} .
$$

Conversely, let $s=\left(D^{*}\right)^{(k+1)} t \in \operatorname{Im}\left(D^{*}\right)^{(k+1)}$. Then, for any $u \in \Gamma_{0}(E)$

$$
(u, s)=\left(u,\left(D^{*}\right)^{(k+1)} t\right)=(D u, t)=0 .
$$


This implies our assertion.

REMARK. (i) In particular, $\operatorname{Im} D^{*}=\lim \left(D^{*}\right)^{(k+1)} \quad($ resp. $\operatorname{Im} D$ $\left.\longleftarrow D^{(k+1)}\right)$ is a closed subspace in $\Gamma(E)$ (resp. in $\Gamma(F)$ ).

(ii) $\Gamma_{0}(E)=\operatorname{Ker} D, \Gamma_{0}(F)=\operatorname{Ker} D^{*}$.

\section{\$4. Main theorems}

In this section, we let $E$ and $F$ denote $C^{\infty}$-fiber bundles over $M$, and let $L$ be a (non-linear) differential operator of $m$-th order from $\Gamma(E)$ to $\Gamma(F)$ which is elliptic at $s \in \Gamma(E)$, namely, its linearization $d_{s} L$ is an elliptic operator from $\Gamma\left(T_{s} E\right)$ to $\Gamma\left(T_{L(s)} F\right)$.

Since the linearization means, roughly speaking, to replace locally the non-linear mapping $L$ by an approximating linear mapping, and further we know that the dimension of the solution space of a linear elliptic equation is finite. We may therefore expect that the dimension of the solution space of any non-linear elliptic equations oughts to be finite. In order to make exact the formulations, we now introduce some notations :

$$
\begin{aligned}
\mathfrak{S}_{s} & =\{t \in \Gamma(E) ; L(t)=L(s)\}, \\
T_{s}\left(\Im_{)}\right) & =\left\{u \in \Gamma\left(T_{s} E\right) ; d_{s} L(u)=0\right\} .
\end{aligned}
$$

We regard $\mathfrak{S}_{s}$ as a topological subspace of $\Gamma(E)$ with the induced topology. It is here noticed that the dimension of $T_{s}(\widetilde{S})$ is finite.

THEOREM 1. Let $L$ be a (non-linear) differential operator which is elliptic at $s \in \Gamma(E)$. Then the solution space $\mathfrak{S}_{s}$ is locally a finite dimensional subset in $\Gamma(E)$ near $s$. More precisely, there is a neighborhood $\mathfrak{H}$ of $s$ in $\Gamma(E)$ with respect to the $C^{\infty}$-topology such that $\mathfrak{S}_{s} \cap \mathfrak{U}$ is diffeomorphic to a locally closed set in finite dimensional vector space $T_{s}(\Im)$.

REMARK. (i ) Even if $L$ is elliptic at any $s \in \Gamma(E)$, the solution space is not in general globally of finite dimension.

(ii) In order to show that the solution space has more fine structures (for example, manifold structure, analytic space structure), we need to suppose more precise assumption of $L: \Gamma(E) \rightarrow \Gamma(F)$ (see, $\S 7$ ).

(iii) Though $L$ is overdetermined elliptic at $s \in \Gamma(E)$, the above assertion is also valid. 
Return now to the examples in $\S 2$. In the first example, we put $L(u)=\Delta u+u+u^{2}$. Thus,

$$
\begin{aligned}
\widetilde{S}_{0} & =\left\{u \in C^{\infty}\left(\boldsymbol{T}^{n}\right) ; \Delta u+u+u^{2}=0\right\}, \\
T_{0}(\widetilde{S}) & =\left\{u \in C^{\infty}\left(\boldsymbol{T}^{n}\right) ; \Delta u+u=0\right\} .
\end{aligned}
$$

Hence, the solution space $\Im_{0}$ must be, by the above theorem, locally realized in $T_{0}(\widetilde{S})$, which is the eigen space of $\Delta$ corresponding to -1 . We make expand $u \in C^{\infty}\left(\boldsymbol{T}^{n}\right)$ in Fourier series:

$$
u(x)=\sum_{\xi \in \mathbb{Z}^{n}} c_{\xi} e^{\sqrt{-1}\langle x, \xi\rangle},
$$

where $\langle x, \xi\rangle=x_{1} \xi_{1}+\cdots+x_{n} \xi_{n}$. If $u \in T_{0}(\widetilde{S})$, then $u$ is expressed by

$$
u(x)=u\left(x_{1}, \cdots, x_{n}\right)=\sum_{i=1}^{n} c_{i} e^{\sqrt{-1} x_{i}}+\sum_{i=1}^{n} c_{-i} e^{-\sqrt{-1} x_{i}},
$$

where $c_{ \pm i}(i=1, \cdots, n)$ are arbitrary complex numbers. From this point of view, we shall parametrize $T_{0}(\subseteq)$ by the coordinate $\left(c_{1}, c_{-1}, \cdots, c_{n}, c_{-n}\right)$. Then, the solution space $\widetilde{S}_{0}$ is realized in $T_{0}(\mathbb{S})$ as follows:

$$
\left\{\left(c_{1}, c_{-1}, \cdots, c_{n}, c_{-n}\right) \in T_{0}\left(S_{)}\right) c_{1} c_{-1}=\cdots=c_{n} c_{-n}=0\right\} \text {. }
$$

Hence, $\widetilde{S}_{0}$ is an analytic set with singularity (in fact, it consists of the $2^{n}$-union of $n$-planes). Especially, the solution $u(x)$ parametrized by $\left(c_{1}, 0, \cdots, c_{n}, 0\right)$ is expressed by

$$
\begin{gathered}
u(x)=\sum_{\xi \geq 0 \xi \in \mathbf{Z}^{n}} k_{\xi} c^{\xi} e^{\sqrt{-1}\langle x, \xi\rangle} \\
\left(c^{\xi}=c_{1}^{\xi_{1}} \cdots c_{n}^{\xi_{n}}\right),
\end{gathered}
$$

where $k_{\xi}$ can be determined by inductive process.

Next, in the second example, the solution space $\widetilde{S}_{\mathrm{Id}}=\left\{\varphi \in C^{\infty}(M, M)\right.$; $\left.L(\varphi)=\varphi^{*} g=g\right\}$ containing the identity mapping is no other than the isometry group of Riemannian manifold $(M, g)$, and $T_{\mathrm{Id}}(\widetilde{S})=\{X \in \Gamma(T M)$; $\left.d_{\mathrm{Id}} L(X)=\mathscr{L}_{x} g=0\right\}$ is the Lie algebra of Killing vector fields, which is identified with the Lie algebra of the isometry group. As is well-known, $\widetilde{S}_{I d}$ is a (finitely dimensional) compact Lie group. Therefore, in this case we can know the global structure of the solution space.

\section{§ 5. Bifurcation}

We keep the notations of previous sections. In this section, we shall construct the bifurcation operator ${ }^{(* * *)}$ associated with $L$ in order to prove (***) We refer to as for this concept the lecture note due to L. Nirenberg: Func-
tional Analysis, Courant Instituts of Mathematical Science, New York Univ., 1960/1961. 
Theorem 1. Without loss of generality we can assume that $E$ and $F$ are $C^{\infty}$-vector bundles over $M$, and $s$ and $L(s)$ are zero sections of $\Gamma(E)$ and $\Gamma(F)$ respectively, because the statement of Theorem 1 is local in its nature, and if necessary we can choose vector bundle neighborhoods. From this view point, we put

$$
\begin{aligned}
\widetilde{S}_{0} & =\{t \in \Gamma(E) ; L(t)=0\}, \\
T_{0}\left(\Im_{)}\right) & =\left\{u \in \Gamma(E) ; d_{0} L(u)=0\right\},
\end{aligned}
$$

and we put $D=d_{0} L$ for the sake of simplicity. From our assumption, $D$ is a linear elliptic operator.

Applying the theorem of Hodge-Kodaira to the elliptic operator $D$, stated in $\S 3$, we have the direct decompositions:

$$
\begin{aligned}
& \Gamma(E)=T_{0}(\widetilde{S}) \oplus \operatorname{Im} D^{*}, \\
& \Gamma(F)=\operatorname{Ker} D^{*} \oplus \operatorname{Im} D,
\end{aligned}
$$

which are orthogonal with respect to the $L^{2}$-inner products.

We put furthermore

$$
\begin{gathered}
H=H_{E}: \Gamma(E) \rightarrow T_{0}(\subseteq), \\
K=G_{F} \square_{F}: \Gamma(F) \rightarrow \operatorname{Im} D=\sum_{\lambda>0} P_{F, \lambda}^{0} .
\end{gathered}
$$

Since $\operatorname{Im} D$ and $\operatorname{Im} D^{*}$ are closed subspaces of the Fréchet spaces, these are also Fréchet spaces with the induce topology. By $T_{0}(\Im) \oplus \operatorname{Im} D$, we mean the direct sum of Fréchet spaces $T_{0}(\widetilde{S})$ and $\operatorname{Im} D$. Then, we define the non-linear operator which we want to call the bifurcation operator:

$$
\Phi: \Gamma(E) \rightarrow T_{0}(\Im) \oplus \operatorname{Im} D
$$

by putting $\Phi(t)=H(t) \oplus K \circ L(t)$. Notice that $\Phi(0)=(0,0)$.

LEMMA 5. The linearization $d_{0} \Phi: \Gamma(E) \rightarrow T_{0}(\Im) \oplus \operatorname{Im} D$ is an isomorphism of Fréchet spaces.

Proof. From the definition of $\Phi$, we have

$$
d_{0} \Phi(u)=H(u) \oplus K \circ d_{0} L(u)
$$

Since $K$ is the projection onto $\operatorname{Im} D$, it follows that

$$
d_{0} \Phi(u)=H(u) \oplus D(u)
$$

We first prove that $d_{0} \Phi$ is surjective. Indeed, let $w=w_{1} \oplus w_{2} \in T_{0}(\Im) \oplus$ 
$\operatorname{Im} D$. We put $u=w_{1}+D^{*} G_{F} w_{2} \in \Gamma(E)=T_{0}(\widetilde{S}) \oplus \operatorname{Im} D^{*}$. If we use the fact that $H D^{*}=0, D D^{*} G_{F} w_{2}=w_{2}-H_{F} w_{2}=w_{2}$, and $D w_{1}=0$, we have

$$
d_{0} \Phi(u)=H w_{1}+H D^{*} G_{F} w_{2}+D w_{1}+D D^{*} G_{F} w_{2}=w_{1}+w_{2},
$$

so that $d_{0} \Phi(u)=w$. In order that $d_{0} \Phi$ is injective, we assume that $H(u)=0$ and $D(u)=0$. According to Corollary 2, in $\S 3$, we have $u=H u+G \square u=0+G D^{*} D u=0$. This shows that $d_{0} \Phi$ is injective. By the open mapping theorem in Fréchet space, $d_{0} \Phi$ gives an isomorphism.

THEOREM 2. $\Phi$ maps a neighborhood $\mathfrak{U}$ of 0 in $\Gamma(E)$ diffeomorphically onto a neighborhood of $\Phi(0)=(0,0)$.

So as to prove this theorem, we shall utilize a slight variation of the so-called implicit function theorem (I.F.T.), which will be discussed in the later section.

As was noted, the usual I.F.T. for Banach spaces asserts that if $\varphi$ is a $C^{1}$-mapping (in the sense of Fréchet derivative) of a Banach space $X$ into a Banach space $Y$, if $\varphi(0)=0$, and if $d_{0} \varphi$ is isomorphic, then $\varphi$ gives a local diffeomorphism around the zero point. The proof is quite elementary, and we use intrinsically the fact that $X$ and $Y$ are Banach spaces. In our case, however, $\Gamma(E)$ and $T_{0}(\mathcal{S}) \oplus \operatorname{Im} D$ are considered as Fréchet spaces with the $C^{\infty}$-topology, and I.F.T. for Fréchet spaces is not in general valid (cf. [2]). Therefore, Lemma 5 does not immediately imply Theorem 2. Under such circumstances, we need to use the more precise culculus of the bifurcation operator $\Phi$ in the Sobolev space $H^{k}$ so as to prove Theorem 2 (see, §6).

By using a standard bifurcation method of non-linear functional analysis, the proof of Theorem 1 can be derived from this theorem, as follows: In fact, let $\mathfrak{U}, \mathfrak{B}_{1}$, and $\mathfrak{B}_{2}$ be neighborhoods respectively in $\Gamma(E), T_{0}(\widetilde{S})$, and $\operatorname{Im} D$ such that $0 \in \mathfrak{H}, 0 \in \mathfrak{B}_{1}, 0 \in \mathfrak{B}_{2}$, and the mapping $\Phi: \mathfrak{U} \rightarrow \mathfrak{B}_{1} \oplus \mathfrak{B}_{2}$ is diffeomorphic. Then a $C^{\infty}$-mapping $Q: \mathfrak{B}_{1} \oplus \mathfrak{B}_{2} \rightarrow$ Ker $D^{*} \oplus \operatorname{Im} D=\Gamma(F)$ can be defined such that in the diagram :

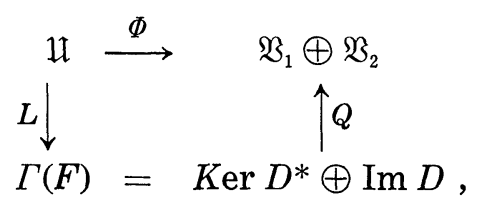

the commutative relation $Q \Phi=L$ is valid. If we define the mapping: 


$$
R: \mathfrak{B}_{1} \oplus \mathfrak{B}_{2} \rightarrow \operatorname{Ker} D^{*}
$$

by $R=H_{F} L \Phi^{-1}$ (as was noted before $H_{F}$ denotes the harmonic projection : $\left.\cdot \Gamma(F) \rightarrow \operatorname{Ker} D^{*}\right)$, we obtain

$$
Q\left(u_{1}, u_{2}\right)=R\left(u_{1}, u_{2}\right) \oplus u_{2},
$$

where $u_{i} \in \mathfrak{B}_{i}(i=1,2)$. Indeed,

$$
Q\left(u_{1}, u_{2}\right)=H_{F} L \Phi^{-1}\left(u_{1}, u_{2}\right) \oplus K L \Phi^{-1}\left(u_{1}, u_{2}\right),
$$

and if we put $\Phi u=u_{1} \oplus u_{2}$, then $u_{2}=K \circ L(u)$. Hence, the above $Q\left(u_{1}, u_{2}\right)$ is equal to $R\left(u_{1}, u_{2}\right) \oplus u_{2}$.

Thus it follows at once that under the diffeomorphism $\Phi, \widetilde{S}^{\prime}=\{(u, 0)$ $\left.\in \mathfrak{B}_{1} \oplus \mathfrak{B}_{2} ; R(u, 0)=0\right\}$ is diffeomorphically mapped onto $\mathfrak{S}_{0} \cap \mathfrak{H}$. This proves that $\mathfrak{S}_{0} \cap \mathfrak{U}$ is diffeomorphic to the set of zeros of finitely many $C^{\infty}$ functions defined on an open set $\mathfrak{B}_{1}$ of the finite dimensional vector space $T_{0}\left(S_{)}\right.$; namely that of zeros of the mapping

$$
R(\cdot, 0): \mathfrak{B}_{1} \rightarrow \operatorname{Ker} D^{*} .
$$

(Note that $\operatorname{Ker} D^{*}$ is of finite dimension).

REMARK. When $L$ is an overdetermined elliptic system at $s$, namely the leading symbol $\sigma_{m}\left(d_{s} L\right)$ is injective, $\operatorname{Ker} D^{*}$ is in general an infinitely dimensional vector space. But, the assertion of Theorem 1 is valid also in this case by similar arguments as above.

COROLlaRY. If $d_{s} L: \Gamma\left(T_{s} E\right) \rightarrow \Gamma\left(T_{L(s)} F\right)$ is surjective, the solution space $\mathfrak{S}_{s}$ has a manifold structure of finite dimension around $s$ with the tangent space $T_{s}(\Im)$ at $s$.

Proof. Rince Ker $D^{*}=0, R(\cdot, 0)$ is the constant 0-mapping. Hence, $\mathfrak{S}_{0} \cap \mathfrak{U}$ id diffeomorphic to an open neighborhood of $T_{0}(\overleftarrow{S})$ q.e.d.

\section{\$6. The Proof of Theorem 2}

This section is devoted to the proof of Theorem 2, keeping the situation of previous sections.

We regard the Fréchet spaces which we consider in this section as the inverse limits of Hilbert spaces:

$$
\begin{aligned}
\Gamma(E) & =\lim H^{k}(E), & \Gamma\left(F^{\prime}\right) & =\lim _{\longleftarrow} H^{k}\left(F^{\prime}\right), \\
\operatorname{Im} D & =\lim \operatorname{Im} D^{(k)}, & \operatorname{Im} D^{*} & =\lim \operatorname{Im}\left(D^{*}\right)^{(k)} .
\end{aligned}
$$


Now we consider the extension of $D=d_{0} L$

$$
D^{(k)}=\left(d_{0} L\right)^{(k)}: H^{k}(E) \rightarrow H^{k-1}\left(F^{\prime}\right),
$$

and the orthogonal decomposition of $H^{k}$ :

$$
\begin{aligned}
H^{k}(E) & =T_{0}(\Im) \oplus \operatorname{Im}\left(D^{*}\right)^{(k+1)}, \\
H^{k-1}(F) & =\operatorname{Ker} D^{*} \oplus \operatorname{Im} D^{(k)} .
\end{aligned}
$$

As is noticed of in $\S 3$, their decompositions are orthogonal with respect to the $L^{2}$-inner product, too. Then we have

LEMMA 6. The restriction of $D^{(k)}$

$$
D^{(k)} \mid \operatorname{Im}\left(D^{*}\right)^{(k+1)}: \operatorname{Im}\left(D^{*}\right)^{(k+1)} \rightarrow \operatorname{Im} D^{(k)}
$$

is an isomorphism of Hilbert spaces.

Proof. First, we note that the restriction is surjective. For, let $u \in \operatorname{Im} D^{(k)}$. Then, there is $w \in H^{k}(E)$ with $D^{(k)} w=u$. Writing

$$
w=w_{1}+w_{2} \in T_{0}(\widetilde{S}) \oplus \operatorname{Im}\left(D^{*}\right)^{(k+1)}
$$

we see that $u=D^{(k)} w=D w_{1}+D^{(k)} w_{2}=D^{(k)} w_{2}$. So, $D^{(k)} \mid \operatorname{Im}\left(D^{*}\right)^{(k+1)}$ is surjective. That $D^{(k)} \mid \operatorname{Im}\left(D^{*}\right)^{(k+1)}$ is injective is obvious from $\operatorname{Ker} D \cap$ $\operatorname{Im}\left(D^{*}\right)^{(k+1)}=0$.

q.e.d.

Next, we define the non-linear operator of Hilbert spaces:

$$
\Phi_{k}: H^{k}(E) \rightarrow T_{0}(\widetilde{S}) \oplus \operatorname{Im} D^{(k)}
$$

by putting $\Phi_{k}(t)=H^{(k)}(t) \oplus K^{(k-1)} \circ L^{(k)}(t)$ for $t \in H^{k}(E)$. Here, we suppose that $k \geqq k_{0}=(1 / m)(m+[n / 2]+1)$.

Proposition 2. The mapping $\Phi_{k}$ is $C^{\infty}$ in a sense of Fréchet derivative, and is an extension of $\Phi$ defined in $\S 5$. Moreover, for $\ell \geqq k$, we have

$$
\Phi_{k} \mid H^{\ell}(E)=\Phi_{\ell}
$$

and $d_{0} \Phi_{k}: H^{k}(E) \rightarrow T_{0}(\mathbb{S}) \oplus \operatorname{Im} D^{(k)}$ is an isomorphism.

REMARK. Namely, $\left\{\Phi_{k}\right\}_{k \geqq k_{0}}$ is an I.L.H.-mapping of I.L.H.-spaces [7].

Proof. The first half of Proposition follows at once from the definition of $\Phi_{k}$ if we note that the direct decompositions are common to any $H^{k}$. The last statement follows from the above lemma and an 
argument that is similar to the proof of Lemma 4 in $\S 5$.

q.e.d.

Hence, if we apply the usual implicit function theorem in Banach spaces to the mapping $\Phi_{k}$, we get

Proposition 3. $\Phi_{k}$ maps a neighborhood $\mathfrak{H}_{k}$ of 0 in $H^{k}(E)$ diffeomorphically onto a neighborhood $\mathfrak{B}_{k-1}$ of $\Phi(0)=(0,0)$ in $T_{0}(\Im) \oplus \operatorname{Im} D^{(k)}$.

This proposition, however, does not immediately lead to our desired results, because we don't know whether we can take in general a sequence $\left\{\mathfrak{H}_{k}\right\}_{k \geqq k_{0}}$ such that

$$
H^{\ell}(E) \cap \mathfrak{H}_{k}=\mathfrak{H}_{\ell}
$$

for $\ell \geqq k$. But, if we make use of a result of A. Douglis and L. Nirenberg [1], we obtain the following weak form:

Proposition 4. There exists a sequence of neighborhood $\left\{\mathfrak{H}_{k}\right\}_{k_{\geqq} k_{0}}$ of 0 in $H^{k}(E)$ and $\left\{\mathfrak{B}_{k-1}\right\}_{k \geqq k_{0}}$ of $\Phi(0)=(0,0)$ in $T_{0}(\widetilde{S}) \oplus \operatorname{Im} D^{(k)}$ such that

(i) $u_{k}: \mathfrak{U}_{k} \rightarrow \mathfrak{B}_{k-1}$ is a diffeomorphism for $k \geqq k_{0}$.

(ii) For $\ell, k \geqq k_{0}$, we have

$\Gamma(E) \cap \mathfrak{U}_{k}=\Gamma(E) \cap \mathfrak{U}_{\ell}, \quad\left(T_{0}(\Im) \oplus \operatorname{Im} D\right) \cap \mathfrak{B}_{k-1}=\left(T_{0}(\Im) \oplus \operatorname{Im} D\right) \cap \mathfrak{B}_{\ell-1}$. where as before, $k_{0}=(1 / m)(m+[n / 2]+1)$.

Before we proceed to prove this proposition, we define the notion of elliptic element of $L$ as follows: Up to the present, we have considered the linearization of $L$ only at the $C^{\infty}$-section $s \in \Gamma(E)$. Of course, for any $s \in H^{k_{0}}(E)$, we can always define the Gâteaux derivative (= Fréchet derivative) $d_{s} L$ at $s$, which is a linear differential operator possibly with the lower differentiable coefficients. In this case too, we can define the leading symbol of $d_{s} L$ as a continuous homomorphism

$$
p^{-1} E \rightarrow p^{-1} F
$$

and if this homomorphism is bijective, we call a cross section $s \in H^{k_{0}}(E)$ an elliptic element of $L$. As a matter of fact, if $L$ is elliptic at $s \in \Gamma(E)$, then $s$ is an elliptic element of $L$.

For the proof of the above statement, we need the following lemma about the $C^{\infty}$-regularity of solutions of a non-linear differential equation of elliptic type, which is due to A. Douglis and L. Nirenberg [1] (see 
Theorem 5):

LEMMA 7. Let $u \in H^{k_{0}}(E)$ be an elliptic element of $L$. We assume that $L^{\left(k_{0}\right)} u$ is a $C^{\infty}$-section of $F$. Then, $u \in \Gamma(E)$.

After these preliminary remarks, let us turn to the proof of Proposition 4. Let $\mathfrak{H}_{k_{0}}$ and $\mathfrak{B}_{k_{0-1}}$ be open neighborhoods such that $\mathfrak{H}_{k_{0}}$ consists of elliptic elements of $L$ and $\Phi_{k_{0}}: \mathfrak{H}_{k_{0}} \rightarrow \mathfrak{H}_{k_{0-1}}$ is a diffeomorphism. (We notice that the set elliptic elements of $L$ is open in $H^{k_{0}}$ ). Then it is enough to show that for $k \geqq k_{0}$ we can choose open neighborhoods $\mathfrak{H}_{k}$, $\mathfrak{B}_{k-1}$ so that $\Phi_{k}: \mathfrak{H}_{k} \rightarrow \mathfrak{B}_{k-1}$ is diffeomorphic, and

$$
\begin{aligned}
\Gamma(E) \cap \mathfrak{H}_{k} & =\Gamma(E) \cap \mathfrak{H}_{k_{0}}, \\
\left(T_{0}(\widetilde{S}) \oplus \operatorname{Im} D\right) \cap \mathfrak{B}_{k-1} & =\left(T_{0}(\Im) \oplus \operatorname{Im} D\right) \cap \mathfrak{B}_{k_{0}-1} .
\end{aligned}
$$

We observe first that for any $t \in \Gamma(E) \cap \mathfrak{H}_{k_{0}}$, there is a neighborhood $\mathfrak{H}_{k}(t)$ of $t$ in $H^{k}(E)$ such that $\Phi_{k}$ is a local diffeomorphism of $\mathfrak{H}_{k}(t)$ onto $\Phi_{k}\left(\mathfrak{U}_{k}(t)\right)$. For this purpose, we show that the linearization

$$
d_{t} \Phi_{k}: H^{k}(E) \rightarrow T_{0}(\Im) \oplus \operatorname{Im} D^{(k)}
$$

is an isomorphism of Hilbert spaces. From the injectivity of $d_{t} \Phi_{k_{0}}$, it is clear that a homomorphism $d_{t} \Phi_{k}$ is also injective. To indicate the surjectivity, let $\left(u_{1}, u_{2}\right) \in T_{0}(\mathfrak{S}) \oplus \operatorname{Im} D^{(k)}$. Then, there is a $\left(w_{1}, w_{2}\right) \in T_{0}$ (S) $\oplus \operatorname{Im}\left(D^{*}\right)^{\left(k_{0}+1\right)}=H^{k_{0}}(E)$ such that $d_{t} \Phi_{k_{0}}\left(w_{1}, w_{2}\right)=\left(u_{1}, u_{2}\right)$, since $\Phi_{k_{0}}$ is a diffeomorphism. In view of the definition of $\Phi_{k_{0}}$, we have

$$
K^{\left(k_{0}-1\right)} d_{t} L^{\left(k_{0}\right)}\left(w_{2}\right)=u_{2},
$$

so

$$
\begin{aligned}
d_{t} L^{\left(k_{0}\right)}\left(w_{2}\right) & =H_{F} d_{t} L^{\left(k_{0}\right)}\left(w_{2}\right)+K^{\left(k_{0}-1\right)} d_{t} L^{\left(k_{0}\right)}\left(w_{2}\right) \\
& =H_{F} d_{t} L^{\left(k_{0}\right)}\left(w_{2}\right)+u_{2} .
\end{aligned}
$$

Since $u_{2} \in \operatorname{Im} D^{(k)}$ and $H_{F} d_{t} L^{\left(k_{0}\right)}\left(w_{2}\right) \in \Gamma(F)$, it follows that $d_{t} L^{\left(k_{0}\right)}\left(w_{2}\right) \in$ $H^{k-1}\left(F^{\prime}\right)$. Hence, according to the $H^{k}$-regularity of linear elliptic operator $d_{t} L$ with $C^{\infty}$-coefficient (we notice that $t \in \Gamma(E)$ ), we have $w_{2} \in H^{k}(E)$ and so $\left(w_{1}, w_{2}\right) \in H^{k}(E)$. This implies that surjectivity of $d_{t} \Phi_{k}$, and by I.F.T., $\Phi_{k}$ gives a local diffeomorphism around $t$.

We put

$$
\mathfrak{H}_{k}=\bigcup_{t} \mathfrak{H}_{k}(t), \quad \mathfrak{B}_{k-1}=\Phi_{k}\left(\mathfrak{H}_{k}\right)
$$


where the union runs over all $t \in \Gamma(E) \cap \mathfrak{H}_{k_{0}}$. Then it is clear that $\mathfrak{H}_{k}$ is an open neighborhooe of 0 in $H^{k}(E), \mathfrak{B}_{k-1}$ an open neighborhood in $H^{k-1}(F)$, and $\Gamma(E) \cap \mathfrak{H}_{k}=\Gamma(E) \cap \mathfrak{H}_{k_{0}}$.

Finally we would like to show that $\left(T_{0}(\subseteq) \oplus \operatorname{Im} D\right) \cap \mathfrak{B}_{k-1}=\left(T_{0}(\subseteq) \oplus\right.$ $\operatorname{Im} D) \cap \mathfrak{B}_{k_{0-1}-}$. Given $u=\left(u_{1}, u_{2}\right) \in\left(T_{0}(\subseteq) \oplus \operatorname{Im} D\right) \cap \mathfrak{B}_{k_{0-1}}$, there is a $w=$ $\left(w_{1}, w_{2}\right) \in \mathfrak{H}_{k_{0}}$ with $\Phi_{k_{0}}(w)=u$. From the similar argument as above, we have

$$
L^{\left(k_{0}\right)} w \in \Gamma\left(F^{\prime}\right) .
$$

Therefore, owing to Lemma 6 we have $w \in \Gamma(E)$, and so $w \in \Gamma(E) \cap \mathfrak{H}_{k_{0}}$. By the definition of $\mathfrak{B}_{k-1}$, we have $u=\Phi_{k}(w) \in \mathfrak{B}_{k-1}$. The inverse inclusion is obvious. This completes the proof of Proposition.

We now put

$$
\begin{aligned}
\mathfrak{H} & =\Gamma(E) \cap \mathfrak{H}_{k_{0}}, \\
\mathfrak{B} & =\left(T_{0}(\mathfrak{S}) \oplus \operatorname{Im} D\right) \cap \mathfrak{B}_{k_{0}-1} .
\end{aligned}
$$

Then, it follows immediately from Proposition 4 that $\Phi: \mathfrak{U} \rightarrow \mathfrak{B}$ gives a diffeomorphism with respect to the Fréchet topology.

\section{§ 7. Final remarks}

In this section, under an additional assumption, we shall state the more precise result as for the solution spaces.

In Theorem 1, we showed that the solution space of an elliptic operator is locally of finite dimension, which is in fact realized as the set of zeros of finitely many $C^{\infty}$-functions on a finite dimensional vector space. In general, the set of zeros of $C^{\infty}$-functions has a complicated structure. We want therefore to seek a sufficient condition so that the solution space has a manifold structure in a local sense.

In the case of smooth mappings, the inverse image of a point is locally a smooth manifold around the point where the mapping has maximal rank. In analogy to the notion of maximal rank of $C^{\infty}$-mapping, we introduce the notion of minimal elements as follows: As cross section $s \in \Gamma(E)$ will be called a locally minimal element of $L$, if $L$ is (overdetermined) elliptic at $s$ and there is an open neighborhood $\mathfrak{U}$ of $s$ such that $\operatorname{dim} T_{s}(\mathbb{S}) \leqq \operatorname{dim} \operatorname{Ker} d_{t} L$ for any $t \in \mathfrak{H}$.

In the elliptic case, index $d_{t} L=\operatorname{dim} \operatorname{Ker} d_{t} L-\operatorname{dim}$ Coker $d_{t} L$ is constant independent on $t \in \mathfrak{H}$ (we assume that $\mathfrak{H}$ is connected), since the 
index of elliptic operator is homotopy invariant. Hence, the above condition is equivalent to:

$$
\operatorname{dim} \text { Coker } d_{t} L \geqq \operatorname{dim} \text { Coker } d_{s} L,
$$

for any $t \in \mathfrak{H}$. In particular, if $d_{s} L$ is onto, $s$ is a locally minimal element.

EXAMPLE. Let $(M, g)$ be a Riemannian manifold. We consider the differential operator introduced in $\S 2$ :

$$
\begin{gathered}
L: C^{\infty}(M, M) \rightarrow \Gamma\left(S^{2} M\right), \\
L(\varphi)=\varphi^{*} g .
\end{gathered}
$$

As is noticed in $\S 2, L$ is overdetermined elliptic at $\operatorname{Id} \in C^{\infty}(M, M)$. Here, we show that Id is a locally minimal element of $L$. For this purpose, we denote by $\mathscr{D}(M)$ the diffeomorphism group of $M$, which is open in $C^{\infty}(M, M)$. We take $\varphi_{1} \in \mathscr{D}(M)$ and let $g_{1}=L\left(\varphi_{1}\right)=\varphi_{1}^{*} g$. We define $L_{1}: C^{\infty}(M, M) \rightarrow \Gamma\left(S^{2} M\right)$ by $L_{1} \varphi=\varphi^{*} g_{1}$. So we have

$$
L_{1} \varphi=\varphi^{*} g_{1}=\varphi^{*} \varphi_{1}^{*} g=\left(\varphi_{1}\right)^{*} g=L R_{\varphi_{1}^{*}}(\varphi)
$$

where $R_{\varphi_{1}}$ is the $C^{\infty}$-fiber bundle mapping (diffeomorphism) defined by $(x, y) \rightarrow\left(x, \varphi_{1}(g)\right)$, namely, $R_{\omega_{1}}: M \times M \rightarrow M \times M$, and $R_{\varphi_{1}^{*}}(\varphi)=\varphi_{1} \varphi$. Therefore $d_{\mathrm{Id}} L_{1}=d_{\varphi_{1}} L d_{\mathrm{Id}} R_{\varphi_{\mathrm{I}}^{*}}$, so that $\operatorname{dim} \operatorname{Ker} d_{\varphi_{1}} L=\operatorname{dim} \operatorname{Ker} d_{\mathrm{Id}} L_{1}=\operatorname{dim} \operatorname{Ker} d_{\mathrm{Id}} L$, since $d_{\mathrm{Id}} R_{\varphi_{1}^{*}}$ is an isomorphism and $\left(M, g_{1}\right)$ is isometric to $(M, g)$ by $\varphi_{1}$. This shows that Id is minimal.

We now return to the first situation. We put $\rho=\operatorname{dim} T_{s}(\widetilde{S})$.

LEMMA 8. Let $s \in \Gamma(E)$ be a locally minimal element of $L$. Then, there is an open neighborhood $\mathfrak{H}^{\prime}(\subset \mathfrak{H})$ of $s$ in $\Gamma(E)$ such that

$$
\rho=\operatorname{dim} T_{s}(\Im)=\operatorname{dim} \operatorname{Ker} d_{t} L
$$

for any $t \in \mathfrak{H}^{\prime}$.

Proof. Let $\mathfrak{U}$ be an open set as in the avove definition. Then $\left\{d_{t} L\right\}_{t \in \mathfrak{u}}$ is a continuous family of elliptic operators over the topological space 1 t. Therefore, by the upper semi-continuity of the dimension of the kernel of an elliptic operator, there is an open neighborhood $\mathfrak{H}^{\prime} \subset \mathfrak{U}$

$$
\begin{aligned}
\rho=\operatorname{dim}\left(T_{s}(\Im)\right) & =\operatorname{dim} \operatorname{Ker}\left(d_{s} L\right) \\
& \geqq \operatorname{dim} \operatorname{Ker}\left(d_{t} L\right)
\end{aligned}
$$

for all $t \in \mathfrak{H}^{\prime}$. This proves the lemma.

q.e.d. 
THEOREM 3. Let $s$ be a locally minimal element of $L$. Then the solution space $\mathfrak{S}_{s}$ is locally a $\rho$-dimensional $C^{\infty}$-manifold around $s$ with the tangent space $T_{s}(\widetilde{S})$ at $s$.

Proof. As the argument in $\S 5$, we assume that $E$ and $F$ are vector bundles and that $s=L(s)=0$ (0-section). Also, we adopt the notations of preceeding sections. We set

$$
\widetilde{S}_{0}^{\prime}=\{t \in \Gamma(E) ; K L(t)=0\} .
$$

Then, $\widetilde{S}_{0}^{\prime}$ is locally a $C^{\infty}$-manifold around the 0 -point, with the tangent space $t+\operatorname{Ker}\left(K d_{t} d\right)$ at $t \in \mathfrak{S}_{0}^{\prime}$, that is an affine subspace of $\Gamma(E)$. For, under the local diffeomorphism $\Phi: \Gamma(E) \rightarrow T_{0}(\widetilde{S}) \oplus \operatorname{Im} D, \Im_{0}^{\prime}$ is diffeomorphic to the open neighborhood of 0 in $T_{0}(\Im)$. We need the following lemma for the proof of Theorem 3 .

LEMMA 9. $H_{F} L \Im_{0}^{\prime} \equiv 0$ around 0 .

Proof. We notice that $H_{F} L: \widetilde{S}_{0}^{\prime} \rightarrow \operatorname{Ker} D^{*}$ is a $C^{\infty}$-mapping of finitely dimensional $C^{\infty}$-manifold $\mathfrak{S}_{0}^{\prime}$. Hence, it is enough to show that $d_{t}\left(H_{F} L \widetilde{S}_{0}\right.$ $=0$ near 0 . Let $u \in T_{t}\left(\Im_{0}^{\prime}\right)=t+\operatorname{Ker}\left(K d_{t} L\right)$. Then, from our assumption, we have

$$
\operatorname{dim} \operatorname{Ker} d_{0} L=\operatorname{dim} \operatorname{Ker} d_{t} L, \text { and } \operatorname{Ker}\left(K d_{t} L\right) \supset \operatorname{Ker} d_{t} L,
$$

so that $\operatorname{Ker}\left(K d_{t} L\right)=\operatorname{Ker} d_{t} L$ and $d_{t}\left(H_{F} L \widetilde{S}_{0}^{\prime}\right)(n)=H_{F} d_{t} L(u)=H_{F}(0)=0$.

Returning to the proof of Theorem 3, we now show that $\widetilde{S}_{0}=\widetilde{S}_{0}^{\prime}$ near 0. From the dfinition, it is obvious that $\mathfrak{S}_{0}^{\prime} \supset \mathbb{S}_{0}$. Take $u \in \mathbb{S}_{0}^{\prime}$ near 0 . Since $H_{F} L u=0$ by Lemma 9 , we have

$$
L u=H_{F} L u+K L u=0 \text {. }
$$

This completes the proof of Theorem 3 .

\section{REFERENCES}

[1] A. Douglis and L. Nirenberg: Interior estimate for elliptic systems of partial differential equations, Comm. Pure Appl. Math., 8 (1955), 503-538.

[2] J. Eells: A setting for Global Analysis, Bull. Amer. Math. Soc., 72 (1966), 751-809.

[ 3 ] M. Kuranishi: New proof for existence of locally complete families of complex structure, Proceeding of the Conference on Complex Analysis, Minneapolis (1964), $142-154$.

[4] J. Moser: A new technique for the construction of solutions of non-linear differential equations, Proc. Nut. Acad. U.S.A., 47 (1961), 1824-1831. 
[5] J. Nash: The imbedding problem for Riemannian manifolds, Ann. Math., 63 (1956), 20-63.

[6 ] L. Nirenberg: The Weyl and Minkowski problems in differential geometry in the large, Comm. Pure Appl. Math., 6 (1953), 337-394.

[7] H. Ômori: On the group of diffeomorphisms on a compact manifold, Proc. Symp. Pure Math., Vol. XV, Amer. Math. Soc. (1970), 167-183.

[8] R. S. Palais: Seminar on the Atiyah Singer index theorem, Princeton study 57 (1965).

[ 9 ] R. S. Palais: Foundation of Global Non-linear Analysis, W. A. Benjamin Inc. (1968).

[10] T. Sunada: The solution spaces of non-linear partial differential equations of elliptic type on compact manifolds, Proc. Japan. Acad., 49 (1973 (, 385-389.

Nagoya University 